\title{
New approach to periodic orbit theory of spectral correlations
}

\author{
Petr Braun, Daniel Waltner \\ Fakultät für Physik, Universität Duisburg-Essen, Lotharstraße 1, 47048 Duisburg, \\ Germany
}

\begin{abstract}
The existing periodic orbit theory of spectral correlations for classically chaotic systems relies on the Riemann-Siegel-like representation of the spectral determinants which is still largely hypothetical. We suggest a simpler derivation using analytic continuation of the periodic-orbit expansion of the pertinent generating function from the convergence border to physically important real values of its arguments. As examples we consider chaotic systems without time reversal as well as the Riemann zeta function and Dirichlet $L$-functions zeros.
\end{abstract}

\section{Introduction}

Application of the random matrix theory (RMT) to the statistics of the energy spectra by Wigner and Dyson was originally motivated by the complexity of spectra of heavy nuclei consisting of many strongly interacting particles. However about 1980 it became clear that quantum systems with just two degrees of freedom also have universal statistical spectral properties described by RMT provided the corresponding classical motion is chaotic, for an overview see Refs. [1, 2, 3, 4]; this statement was formulated as the famous Bohigas-Giannoni-Schmit conjecture [5]. Its understanding took many investigations, to mention but a few relevant here: the diagonal approximation [6], a heuristic derivation of the oscillatory part of the correlation function for the unitary unversality class [7], the orbit partnership role in spectral correlations [8], the semiclassical treatment of spin in the level statistics [9, 10, 11], the periodic orbit theory of the small time form factor 12. The complete semiclassical derivation of the two-point correlation function for the unitary and orthogonal universality classes is given in [13, 14] and for the symplectic class in [15]. Three- and higher-level correlation functions are considered in [16].

The state-of-the-art calculation of spectral correlators is based on the method of generating functions imported from the field theory of disorded media, which are ratios of spectral determinants $\operatorname{det}(E+e-H)$ with slightly different energy offsets $e$ averaged over the central energy $E$. The fundamental role in the calculation is played by the so called Riemann-Siegel lookalike [17, 18]. This is the asymptotic representation of a spectral determinant by an explicitly real sum over sets of periodic orbits, or "pseudoorbits", truncated at pseudo-orbits with total period half the Heisenberg time $T_{H}$. 

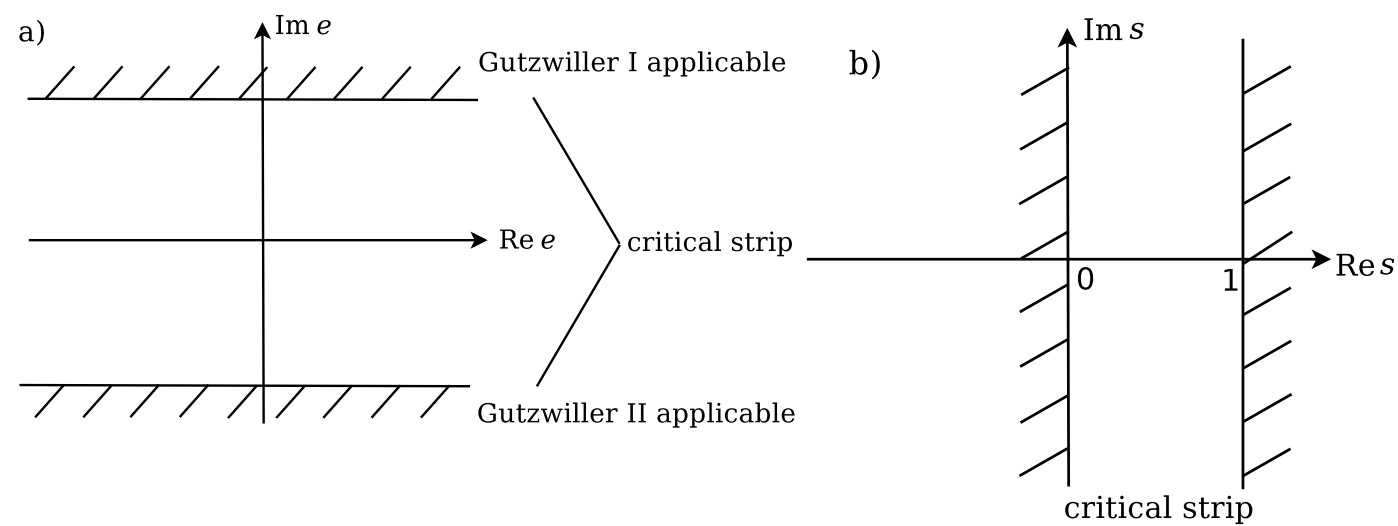

Figure 1. Analogy between the semiclassical periodic orbit theory and the theory of the Riemann zeta function. a) The generating function: two different Gutzwiller expansions are applicable outside the critical strip. b) The Riemann zeta function $\zeta(s)$ case.

The result is far from obvious and has been proven only for some rare cases [19, 20], beyond the Riemann-Siegel formula proper in the theory of the Riemann zeta function [21, 22, 23]. Its usage in problems of spectral statistics looks also like an overkill since the non-trivial boundary $T_{H} / 2$ doesn't play any role; after the averaging, summation over the pseudo-orbits is invariably extended to infinite periods.

Here we want to reformulate the theory in the form which avoids the use of the Riemann-Siegel lookalike. The idea is to complexify the generating function by letting its energy arguments have an imaginary part large enough for convergence of the pertinent Gutzwiller expansions like the Gutzwiller trace formula. It can be done in two ways depending on the sign of the imaginary parts of the energies $e$ which, after averaging, provides two different semiclassical asymptotics which can be analytically continued inside the critical strip. We will refer to these two ways as I and II, for an illustration, see Fig. 1 a). The semiclassical approximation of the generating function in the vicinity of the real energy axis smoothly matching both of these asymptotics is their sum since the "wrong" component is exponentially subdominant for energies with sufficiently large absolute value of the imaginary part.

The paper is built in the following way. In Section 2 we describe the semiclassical periodic orbit theory of the generating function justifying its composition of two parts responsible for the monotonic and oscillatory parts of the level-level correlation function. In Section 3 we remind how the diagonal approximation neglecting the orbit action correlation is introduced for the generating functions; unlike Berry's diagonal approximation it provides both the oscillatory and monotonic parts of the spectral correlator. In Section 4 we show that our approach applied to the spectral correlator of the Riemann zeta function $\zeta(s)$ and Dirichlet $L$-functions zeros reproduces in a more transparent way the results of Bogomolny and Keating [7] and Bogomolny [24]. We apply here arguments similar to the ones used in the case of the periodic orbit theory, for an illustration see Fig. 10). In Section 5 we return to dynamical systems without 
time-reversal symmetry and show how the oscillatory part of the correlator including nonuniversal effects stemming from repetitions can be obtained by our approach. In section 6 we explain why in the presence of time-reversal symmetry the oscillatory part of the correlator cannot be obtained without taking into account periodic orbit action correlations explicitly. Finally we conclude in section 7 .

\section{Correlation function}

The level-level correlation function can be defined as

$$
R(e)=\frac{\left\langle\rho\left(E-\frac{e}{2}\right) \rho\left(E+\frac{e}{2}\right)\right\rangle_{E}}{\left\langle\rho\left(E-\frac{e}{2}\right)\right\rangle_{E}\left\langle\rho\left(E+\frac{e}{2}\right)\right\rangle_{E}}-1
$$

where $\rho(E)$ is the level density; averaging is done over an interval of the reference energy $E$ large compared with the mean level spacing $\delta=1 / \bar{\rho}, \quad \bar{\rho}=\langle\rho\rangle_{E}$, but small in classical terms; $e$ is the energy offset. The non-trivial part of $R(e)$

$$
r(e) \equiv\left\langle\rho\left(E-\frac{e}{2}\right) \rho\left(E+\frac{e}{2}\right)\right\rangle_{E}
$$

to which we shall refer below as the correlation function for brevity, can be expressed in terms of the averaged ratio of four spectral determinants, or the generating function,

$$
Z\left(e_{A}, e_{B}, e_{C}, e_{D}\right)=\left\langle\frac{\operatorname{det}\left(E+e_{C}-H\right) \operatorname{det}\left(E-e_{D}-H\right)}{\operatorname{det}\left(E+e_{A}-H\right) \operatorname{det}\left(E-e_{B}-H\right)}\right\rangle_{E} .
$$

The connection between these quantities is given by the formula,

$$
r(e)=-\left.\frac{1}{2 \pi^{2}} \operatorname{Re} \frac{\partial^{2} Z}{\partial e_{A} \partial e_{B}}\right|_{\|},
$$

assuming that the arguments of $Z$ have imaginary parts of the same sign for $e_{A}, e_{B}$ as well as for $e_{C}, e_{D}$ : otherwise the result of averaging with respect to $E$ would be zero. After the averaging, all $e_{X}, \quad X=A, B, C, D$, must be set to $e / 2$ which is indicated by (II) in (2); this is the so called "columnwise" limit of [13].

Without loss of generality we can fix for example $\operatorname{Im} e_{A, B}>0$ with the limit value $0_{+}$. As regards the energy offsets in the numerator we can then choose either $\operatorname{Im} e_{C, D}>0$ or $\operatorname{Im} e_{C, D}<0$ with the limit values $0_{+}$and $0_{-}$respectively. The two corresponding limits of $Z$ must coincide as the latter quantity is well defined for real $e_{C}$ and $e_{D}$. On the other hand, changing the sign of $\operatorname{Im} e_{C, D}$ is equivalent to the replacement $e_{C} \rightleftarrows-e_{D}$ in $Z$. We come thus to the so called Weyl symmetry relation valid for real $e_{C, D}$,

$$
Z\left(e_{A}, e_{B}, e_{C}, e_{D}\right)=Z\left(e_{A}, e_{B},-e_{D},-e_{C}\right) \quad \text { with } \quad \operatorname{Im} e_{C, D}=0 .
$$

The semiclassical asymptotics of the generating function follows from the Gutzwiller periodic orbit expansion [3] of the trace of the resolvent. It converges when $\operatorname{Im} E$ is positive and large enough (the value guaranteeing absolute convergence is half the Kolmogorov-Sinai entropy, in view of the exponential orbit proliferation) and is given by

$$
\operatorname{Tr}(E-H)^{-1} \sim-i \pi \bar{\rho}(E)-\frac{i}{\hbar} \sum_{p} T_{p} F_{p} \mathrm{e}^{i S_{p}(E) / \hbar} \quad \text { for } \quad \operatorname{Im} E>0
$$


where we neglected the contributions from the orbit repetitions. The classical action of the orbit $p$ is denoted by $S_{p}$, the duration of the periodic orbits $T_{p}$ and the stability prefactor by $F_{p}$, for their precise form see Ref. [3]. The expansion of the spectral determinant then follows from the identity $\operatorname{det}(E-H)=\exp \operatorname{Tr} \ln (E-H)$ which gives,

$$
\begin{aligned}
& \operatorname{det}(E-H) \propto \exp \operatorname{Tr} \int^{E} d E^{\prime}\left(E^{\prime}-H\right)^{-1} \propto \exp [-i \pi \bar{N}(E)] \prod_{p} d_{p}(E), \\
& \text { with } \operatorname{Im} E>0 \text { and } d_{p}(E)=\exp \left[-F_{p} \mathrm{e}^{i S_{p}(E) / \hbar}\right],
\end{aligned}
$$

where

$$
\bar{N}(E)=\int^{E} d E^{\prime} \bar{\rho}\left(E^{\prime}\right)
$$

denotes the averaged level counting function. The spectral determinant with the argument whose imaginary part is negative, is obtained by complex conjugation,

$$
\operatorname{det}(E-H) \propto \exp [i \pi \bar{N}(E)] \prod_{p} d_{p}^{*}(E) \quad \text { with } \quad \operatorname{Im} E<0 .
$$

The two expansions can be used to get the semiclassical approximation of the generating function. Considering that $\operatorname{Im} e_{A, B}>0$ we substitute (44) for $\operatorname{det}\left(E+e_{A}-H\right)$ and (6) for $\operatorname{det}\left(E-e_{B}-H\right)$. As regards the numerator there are two possibilities. If we choose $\operatorname{Im} e_{C, D}>0$ we must use (44) for $\operatorname{det}\left(E+e_{C}-H\right)$ and (6) for $\operatorname{det}\left(E-e_{D}-H\right)$. Expanding $\bar{N}\left(E+e_{A}\right)=\bar{N}(E)+e_{A} \bar{\rho}$ etc. we obtain the semiclassical asymptotics of the determinants ratio as

$$
\begin{gathered}
Z \sim Z^{I}\left(e_{A}, e_{B}, e_{C}, e_{D}\right)=\mathrm{e}^{i \pi\left(e_{A}+e_{B}-e_{C}-e_{D}\right) \bar{\rho}}\left\langle\prod_{p} z_{p}\left(e_{A}, e_{B}, e_{C}, e_{D}\right)\right\rangle, \\
\quad \text { with } \quad \operatorname{Im} e_{C, D}>0 \text { and } \operatorname{Im} e_{A, B}>0
\end{gathered}
$$

with the definition

$$
z_{p}\left(e_{A}, e_{B}, e_{C}, e_{D}\right)=d_{p}\left(E+e_{C}\right) d_{p}^{*}\left(E-e_{D}\right) d_{p}\left(E+e_{A}\right) d_{p}^{*}\left(E-e_{B}\right) .
$$

If we choose $\operatorname{Im} e_{C, D}<0$ we get an alternative expression,

$$
\begin{aligned}
Z & \sim Z^{I I}\left(e_{A}, e_{B}, e_{C}, e_{D}\right)=Z^{I}\left(e_{A}, e_{B},-e_{D},-e_{C}\right)=\mathrm{e}^{i \pi\left(e_{A}+e_{B}+e_{C}+e_{D}\right) \bar{\rho}} \\
& \times\left\langle\prod_{p} z_{p}\left(e_{A}, e_{B},-e_{D},-e_{C}\right)\right\rangle \text { with } \operatorname{Im} e_{C, D}<0 \text { and } \operatorname{Im} e_{A, B}>0 .
\end{aligned}
$$

The functions $Z^{I}, Z^{I I}$ resulting after the energy averaging are well-behaved analytic functions of their arguments in which $\operatorname{Im} e_{A, B}$ can be brought to the limit $0_{+}$whereas $e_{C, D}$ can take any complex value. This is motivated by the fact that the presence of imaginary parts of the energy arguments in the denominator is obligatory in order to avoid the poles; on the other hand $e_{C}$ and $e_{D}$ can take any real values. It is important that due to the prefactors $\exp \left[\mp i \pi\left(e_{C}+e_{D}\right) \bar{\rho}\right]$ the function $Z^{I I}\left(e_{A}, e_{B}, e_{C}, e_{D}\right)$ analytically continued to the "alien" domain with $\operatorname{Im} e_{C, D}$ large and positive, is exponentially smaller than $Z^{I}\left(e_{A}, e_{B}, e_{C}, e_{D}\right)$. Similarly the analytic continuation of $Z^{I}$ to $\operatorname{Im} e_{C}, e_{D}<0$ is exponentially small compared to $Z^{I I}$. With $e_{C, D}$ in the vicinity of the real axis $Z^{I}, Z^{I I}$ are of the same order of magnitude. 
What we are interested in is the semiclassical asymptotics of the generating function for $e_{C, D}$ real. We know that its analytical continuation to $e_{C, D}$ complex tends to $Z^{I}$ for large $\operatorname{Im} e_{C, D}>0$, and to $Z^{I I}$ for $\operatorname{Im} e_{C, D}<0$. Their sum has this property and yields thus the semiclassical asymptotic for $Z$,

$$
Z \sim Z_{\mathrm{sc}}=Z^{I}+Z^{I I}
$$

which uniformly approximates $Z$ for both signs of the imaginary parts and reduces either to $Z^{I}$ or to $Z^{I I}$ when $\left|\operatorname{Im} e_{C, D}\right|$ is large. Like the exact generating function it obeys the Weyl symmetry relation when $e_{C, D}$ are real,

$$
Z_{\mathrm{sc}}\left(e_{A}, e_{B}, e_{C}, e_{D}\right)=Z_{\mathrm{sc}}\left(e_{A}, e_{B},-e_{D},-e_{C}\right) .
$$

\section{Diagonal approximation}

Consider $Z^{I}$ with all its arguments containing large positive imaginary parts safeguarding convergence of the Gutzwiller expansions. Then $Z^{I}$ can be written as the energy average of a converging product over periodic orbits. Expanding the classical actions like $S_{p}\left(E+e_{A}\right)=S_{p}(E)+T_{p} e_{A}$ etc. we can write it as,

$$
Z^{I}\left(e_{A}, e_{B}, e_{C}, e_{D}\right)=\mathrm{e}^{i \pi\left(e_{A}+e_{B}-e_{C}-e_{D}\right) \bar{\rho}}\left\langle\prod_{p} z_{p}\right\rangle_{E},
$$

where

$z_{p}=\exp \left[F_{p} \mathrm{e}^{i S_{p}(E) / \hbar}\left(\mathrm{e}^{i T_{p} e_{A} / \hbar}-\mathrm{e}^{i T_{p} e_{C} / \hbar}\right)+F_{p}^{*} \mathrm{e}^{-i S_{p}(E) / \hbar}\left(e^{i T_{p} e_{B} / \hbar}-e^{i T_{p} e_{D} / \hbar}\right)\right]$.

Neglecting correlations between $z_{p}$ corresponding to orbits which do not have exactly the same action we can replace the average of the product by the product of averages. In the absence of time reversal and spatial symmetry the orbit actions are non-degenerate. Disregarding the periodic orbit repetitions we get,

$$
Z^{I} \approx \mathrm{e}^{i \pi\left(e_{A}+e_{B}-e_{C}-e_{D}\right) \bar{\rho}} \prod_{p}\left\langle z_{p}\right\rangle .
$$

By the analogous steps as in [13] we obtain,

$$
Z^{I} \approx \mathrm{e}^{i \pi\left(e_{A}+e_{B}-e_{C}-e_{D}\right) \bar{\rho}} \tilde{Z}_{G U E}^{I} \quad \text { with } \quad \tilde{Z}_{G U E}^{I}=\frac{\left(e_{A}+e_{D}\right)\left(e_{B}+e_{C}\right)}{\left(e_{A}+e_{B}\right)\left(e_{C}+e_{D}\right)} .
$$

The second component of $Z$ is obtained by the interchange of $e_{C, D}$ and change of their signs (the Weyl symmetry operation),

$$
Z^{I I} \approx \mathrm{e}^{i \pi\left(e_{A}+e_{B}+e_{C}+e_{D}\right) \bar{\rho}} \tilde{Z}_{G U E}^{I I} \quad \text { with } \quad \tilde{Z}_{G U E}^{I I}=-\frac{\left(e_{A}-e_{C}\right)\left(e_{B}-e_{D}\right)}{\left(e_{A}+e_{B}\right)\left(e_{C}+e_{D}\right)} .
$$

The correlation function obtained as the real part of $\left.\partial_{e_{A} e_{B}}^{2}\left(Z^{I}+Z^{I I}\right)\right|_{\|}$coincides with the correlation function of the GUE ensemble, its monotonic and oscillatory parts produced by $Z^{I}$ and $Z^{I I}$, respectively. We observe that the product $\left(e_{A}-e_{C}\right)\left(e_{B}-e_{D}\right)$ in $\tilde{Z}_{G U E}^{I I}$ turns into zero in the columnwise limit $e_{A, B, C, D} \rightarrow e / 2$ such that both differentiations must be spent on it, hence $\left(\partial_{e_{A} e_{B}}^{2} Z^{I I}\right)_{\|}=-\mathrm{e}^{i 2 \pi e \bar{\rho}} / e^{2}$. 
The usual diagonal approximation as introduced by Berry [6] neglects terms corresponding to different orbits in the expansion of the correlation function itself. Only the non-oscillatory part of the correlator is then recovered; the oscillatory part is regarded as the result of the periodic orbits action correlations. It was demonstrated by Keating [25] and Bogomolny [24] that the oscillatory contribution to the correlation function of the Riemann zeta zeros results from the tendency of the prime numbers ("periodic orbits" of the problem) to avoid one another described by the HardyLittlewood conjecture. The respective orbit correlation mechanism [26] in physical systems without time reversal may thus be called "the Hardy-Littlewood lookalike"; its nature is still not quite clear. It is remarkable that we can completely neglect that correlation using instead the diagonal approximation for the Weyl-symmetric generating function, and get the same result. That equivalence seems to hold also for corrections to the universal RMT result; compare the Riemann zeta treatment by Bogomolny [24], Conrey and Snaith [34] and the one below in this paper.

\section{Zeros of Riemann zeta function}

Following the idea of Hilbert and Pólya, the non-trivial zeros of the Riemann zeta function may be interpreted as the eigenvalues of a self-adjoint Hamilton operator of a virtual dynamical system. The analogy between the density of zeros of the Riemann zeta and the Gutzwiller trace formula for chaotic quantum systems provides further support for this spectral interpretation. Another evidence for this interpretation provides the behavior of the pair correlation of the zeros of the Riemann zeta function first studied by Montgomery [27]; they are the same as for the eigenvalues of the Gaussian unitary ensemble of random matrices (GUE) as confirmed by Odlyzko's numerical calculations [28].

The spectral properties of the Riemann zeta function and closely related $L$-functions were a subject of an innumerable number of investigations; in connection with the present paper we would like to mention the works of Keating [25], Bogomolny et al. [7, 24, 29, 30, 31, 32] and Berry et al. [33]. See also Ref. 34] for a review and the references therein. Here we show that the pair correlation function including the system-specific corrections to the GUE prediction can easily be obtained by the method described above.

\subsection{The generating function}

The Riemann zeta function can be defined by the Euler product over primes,

$$
\zeta(s)=\prod_{p} \frac{1}{1-p^{-s}}
$$

which converges provided $\operatorname{Re} s>1$ but can be analytically continued to the whole complex plane. According to the Riemann hypothesis, all non-trivial zeros of zeta have the form $s_{k}=\frac{1}{2}+i E_{k}$ with $E_{k}$ real; the spectrum is symmetric with respect to $E=0$. 
The corresponding spectral determinant $\Delta(E)=\prod_{k} A\left(E, E_{k}\right)\left(E-E_{k}\right)$ where $A\left(E, E_{k}\right)$ stands for a suitable regularizing function, is related to the Riemann zeta function by

$$
\Delta(E)=B(E) e^{-i \pi \bar{N}(E)} \zeta\left(\frac{1}{2}-i E\right)
$$

with $\bar{N}(E)$ being the mean counting function, i.e. the integral of the mean zeros density $\bar{\rho}(E)$ with respect to $E$, and $B(E)$ a smooth function positive for real $E$ whose explicit form depends on the regularization choice [25]. We need only the asymptotic expressions for large $E$ :

$$
\bar{N}(E) \sim \frac{E}{2 \pi}\left(\ln \frac{E}{2 \pi}-1\right), \quad \bar{\rho}(E)=\frac{d \bar{N}}{d E} \sim \frac{1}{2 \pi} \ln \frac{E}{2 \pi} .
$$

The Euler product for the spectral determinant converges for $\operatorname{Im} E>1 / 2$, and then

$$
\Delta(E)=B(E) \mathrm{e}^{-i \pi \bar{N}(E)} \prod_{p} \frac{1}{1-p^{-1 / 2+i E}} .
$$

When $\operatorname{Im} E<-\frac{1}{2}$ the complex conjugation gives

$$
\Delta(E)=B(E) \mathrm{e}^{i \pi \bar{N}(E)} \prod_{p} \frac{1}{1-p^{-1 / 2-i E}} .
$$

Calculating $\frac{d}{d E} \log \Delta(E)$ we would get the Gutzwiller-like formula for the trace of the virtual resolvent with primes as periodic orbits, analogous to (3) and its complex conjugate but taking into account the orbit repetitions. In fact we don't need that to construct the generating function since the expansion for

$$
Z=\left\langle\frac{\Delta\left(E+e_{C}\right) \Delta\left(E-e_{D}\right)}{\Delta\left(E+e_{A}\right) \Delta\left(E-e_{B}\right)}\right\rangle_{E} \quad \text { with } E \text { real, } \operatorname{Im} e_{A, B}>0,
$$

follows directly from the Euler product of $\zeta$. The generating function can be written in two versions. If $\operatorname{Im} e_{C, D}>0$ we use (9) for $\Delta\left(E+e_{C}\right), \Delta\left(E+e_{A}\right)$ and (10) for the two other determinants. Let us assume all deviations $e$ small compared with $E \gg 1$ such that $\bar{N}\left(E+e_{A}\right) \approx \bar{N}(E)+\bar{\rho} e_{A}$ etc. and $\left|\operatorname{Im} e_{X}\right|>1 / 2, \quad X=A, B, C, D$. Denoting

$$
a_{p} \equiv \frac{\mathrm{e}^{i e_{A} \ln p}}{\sqrt{p}}
$$

and similar for $b_{p}, c_{p}, d_{p}$ we have for $\operatorname{Im} e_{A, B, C, D}>1 / 2$,

$$
\begin{aligned}
& Z^{I}\left(e_{A}, e_{B}, e_{C}, e_{D}\right)=\exp \left[-i \pi\left(e_{C}+e_{D}-e_{A}-e_{B}\right) \bar{\rho}\right]\left\langle\prod_{p} z_{p}\right\rangle_{E}, \\
& \quad z_{p}=\frac{\left(1-a_{p} \mathrm{e}^{i E \ln p}\right)\left(1-b_{p} \mathrm{e}^{-i E \ln p}\right)}{\left(1-c_{p} \mathrm{e}^{i E \ln p}\right)\left(1-d_{p} \mathrm{e}^{-i E \ln p}\right)}
\end{aligned}
$$

we neglected the $e$-dependence of the smooth function $B$. The alternative representation $Z^{I I}$ with $\operatorname{Im} e_{C, D}$ negative is obtained from $Z^{I}$ by the Weyl substitution, $Z^{I I}\left(e_{A}, e_{B}, e_{C}, e_{D}\right)=Z^{I}\left(e_{A}, e_{B},-e_{D},-e_{C}\right) \quad$ with $\operatorname{Im} e_{A, B}>1 / 2, \quad \operatorname{Im} e_{C, D}<-1 / 2 ;$ with the substitution rule $\left\{c_{p}, d_{p}\right\} \rightarrow\left\{\left(d_{p} p\right)^{-1},\left(c_{p} p\right)^{-1}\right\}$. 
New approach to periodic orbit theory of spectral correlations

Now we introduce the diagonal approximation by the assumption that $z_{p}$ associated with different primes are uncorrelated such that the energy average of the product of $z_{p}$ can be replaced by the product of their averages writing

$$
Z^{I} \approx \exp \left[-i \pi\left(e_{C}+e_{D}-e_{A}-e_{B}\right) \bar{\rho}\right] \prod_{p}\left\langle z_{p}\right\rangle_{E} .
$$

The factors in the product are elementarily calculated,

$$
\begin{aligned}
\left\langle z_{p}\right\rangle_{E} & =\frac{\ln p}{2 \pi} \int_{0}^{\frac{2 \pi}{\ln p}} d E z_{p}=\frac{1}{2 \pi i} \oint_{|u|=1} \frac{d u}{u} \frac{\left(1-a_{p} u\right)\left(1-b_{p} / u\right)}{\left(1-c_{p} u\right)\left(1-d_{p} / u\right)} \\
& =1+\frac{\left(b_{p}-d_{p}\right)\left(a_{p}-c_{p}\right)}{1-c_{p} d_{p}} .
\end{aligned}
$$

Considering that $\zeta\left(1-i\left(e_{A}+e_{B}\right)\right)=\prod_{p}\left(1-a_{p} b_{p}\right)^{-1}$ etc, we write $Z^{I}=Z_{\zeta}^{I} \Phi^{I}$, where

$Z_{\zeta}^{I}=\exp \left[-i \pi\left(e_{C}+e_{D}-e_{A}-e_{B}\right) \bar{\rho}\right] \frac{\zeta\left(1-i\left(e_{A}+e_{B}\right)\right) \zeta\left(1-i\left(e_{C}+e_{D}\right)\right)}{\zeta\left(1-i\left(e_{C}+e_{B}\right)\right) \zeta\left(1-i\left(e_{A}+e_{D}\right)\right)}$.

The function $\Phi^{I}$ is a product over primes,

$$
\begin{aligned}
\Phi^{I}\left(e_{A}, e_{B}, e_{C}, e_{D}\right) & =\prod_{p} \phi_{p}^{I}, \\
\text { with } \quad \phi_{p}^{I} & =\frac{\left(1-a_{p} b_{p}\right)\left(1-c_{p} d_{p}\right)}{\left(1-b_{p} c_{p}\right)\left(1-a_{p} d_{p}\right)}\left\langle z_{p}\right\rangle=1-\frac{a_{p} b_{p}\left(a_{p}-c_{p}\right)\left(b_{p}-d_{p}\right)}{\left(1-b_{p} c_{p}\right)\left(1-a_{p} d_{p}\right)} .
\end{aligned}
$$

The second component $Z^{I I}$ is obtained by the Weyl substitution $e_{C} \rightleftarrows-e_{D}$ and can be written as $Z_{\zeta}^{I I} \Phi^{I I}$ with

$Z_{\zeta}^{I I}=\exp \left[-i \pi\left(-e_{C}-e_{D}-e_{A}-e_{B}\right) \bar{\rho}\right] \frac{\zeta\left(1-i\left(e_{A}+e_{B}\right)\right) \zeta\left(1+i\left(e_{C}+e_{D}\right)\right)}{\zeta\left(1-i\left(e_{B}-e_{D}\right)\right) \zeta\left(1-i\left(e_{A}-e_{C}\right)\right)}$

and $\Phi^{I I}\left(e_{A}, e_{B}, e_{C}, e_{D}\right)=\Phi^{I}\left(e_{A}, e_{B},-e_{D},-e_{C}\right)$.

Considering the exponential factors in $Z_{\zeta}$ the sum

$$
Z \sim Z^{I}+Z^{I I}
$$

will smoothly interpolate between $Z^{I}$ and $Z^{I I}$ as we change $\operatorname{Im} e_{C, D}$ from positive to negative values, providing thus the high-energy asymptotics of the generating function for real $e_{C, D}$. It coincides with the result obtained differently in [34].

\subsection{The correlation function}

The correlation function based on Eq. (15) consists of two summands. Differentiation of $Z^{I}$ with the subsequent columnwise identification $(\|) e_{A}=e_{B}=e_{C}=e_{D}=e / 2$ gives the contribution which Bogomolny [24] refers as the diagonal one. It is easily checked that

$$
\left(Z_{\zeta}^{I}\right)_{\|}=1, \quad\left(\frac{\partial Z^{I}}{\partial e_{A}}\right)_{\|}=\left(\frac{\partial Z^{I}}{\partial e_{B}}\right)_{\|}=0
$$

and for the second derivative

$$
\left(\frac{\partial^{2} Z_{\zeta}^{I}}{\partial e_{A} \partial e_{B}}\right)_{\|}=\frac{\partial^{2} \ln \zeta(1-i e)}{\partial e^{2}}=\frac{1}{e^{2}}+\left(\gamma_{0}^{2}+2 \gamma_{1}\right)+O(e) .
$$


For $\Phi^{I}$ we obtain

$$
\Phi_{\|}^{I}=1, \quad\left(\frac{\partial \Phi^{I}}{\partial e_{A}}\right)_{\|}=\Phi_{\|}^{I} \sum_{p}\left(\frac{1}{\phi_{p}^{I}} \frac{\partial \phi_{p}^{I}}{\partial e_{A}}\right)_{\|}=0
$$

and the same result for $\left(\partial \Phi^{I} / \partial e_{B}\right)_{\|}$. For the second derivatives we get

$$
\left(\frac{\partial^{2} \Phi^{I}}{\partial e_{A} \partial e_{B}}\right)_{\|}=\left(\sum_{p} \frac{\partial}{\partial e_{B}}\left(\frac{1}{\phi_{p}^{I}} \frac{\partial \phi_{p}^{I}}{\partial e_{A}}\right)\right)_{\|}=\sum_{p} \frac{\ln ^{2} p}{\left(1-p^{1-i e}\right)^{2}} .
$$

In total we get for the contribution to the spectral correlation function (1)

$$
r^{I}(\varepsilon)=-\frac{1}{2 \pi^{2}} \operatorname{Re}\left(\frac{\partial^{2} Z_{\zeta}^{I}}{\partial e_{A} \partial e_{B}}\right)_{\|}-\frac{1}{2 \pi^{2}} \operatorname{Re}\left(\frac{\partial^{2} \Phi^{I}}{\partial e_{A} \partial e_{B}}\right)_{\|} .
$$

Taking into account the relations (16][17],18],19), we confirm that this result is identical to the "diagonal" one obtained by Bogomolny from the Hardy-Littlewood conjecture in [24].

The second contribution to the spectral correlation function, termed conventionally off-diagonal but which we obtain remaining in the framework of uncorrelated primes, is provided by the second derivative of $Z^{I I}$ with respect to $e_{A}$ and $e_{B}$ and columnwise identification. This identification implies that $e_{A}-e_{C}$ and $e_{B}-e_{D}$ go to zero, hence the following expansion for the inverse zeta functions entering $Z_{\zeta}^{I I}$ can be approximated by

$$
\zeta^{-1}\left(1-i\left(e_{A}-e_{C}\right)\right) \zeta^{-1}\left(1-i\left(e_{B}-e_{D}\right)\right) \approx-\left(e_{A}-e_{C}\right)\left(e_{B}-e_{D}\right)
$$

such that the only term surviving within this identification results from $\partial^{2} Z_{\zeta}^{I I} / \partial e_{A} \partial e_{B}$ yielding $-|\zeta(1-i e)|^{2}$. We get in total for the oscillatory ("off-diagonal") contribution to the correlator,

$$
r^{I I}(e)=\frac{1}{2 \pi^{2}}|\zeta(1-i e)|^{2} \operatorname{Re}\left[\mathrm{e}^{2 \pi i \varrho e} \prod_{p}\left(1-\frac{\left(1-p^{i e}\right)^{2}}{(p-1)^{2}}\right)\right],
$$

where the product in the last equation results from $\left(\Phi^{I I}\right)_{\|}=\Phi^{I}(e / 2, e / 2,-e / 2,-e / 2)$ with $\Phi^{I}$ given by Eq. (13). Again we reproduced the result of Bogomolny and Keating obtained in Ref. [7] in a heuristic way.

\subsection{L-functions}

$L$-functions are generalizations of the Riemann zeta function

$$
L(s)=\sum_{n=1}^{\infty} \frac{\chi(n)}{n^{s}}=\prod_{p} \frac{1}{1-\frac{\chi(p)}{p^{s}}}
$$

including the Dirichlet character $\chi(n)$ defined as a function on integers periodic with the integer period $k$ such that $\chi(n+k)=\chi(n)$, multiplicative $\chi(n) \chi(m)=\chi(n m)$, equal to zero iff the greatest common divisor of $n$ and $k$ is greater than one. We restrict our considerations to real characters $\chi(n)= \pm 1,0$. The generalized Riemann hypothesis states that all non-trivial zeros of $L(s)$ lie on the critical line $s=1 / 2+i E$. The correlation function of these zeros can be determined using the generating function in a 
similar way as for the Riemann zeta function starting from the product representation given in Eq. (23). The generating function obtained under the assumption $\operatorname{Im} e_{A, B, C, D}>$ 0 differs from the Riemann zeta case by the presence of characters in the factors $z_{p}$; these are now

$$
z_{p}=\frac{\left(1-\chi(p) a_{p} \mathrm{e}^{i E \ln p}\right)\left(1-\chi(p) b_{p} \mathrm{e}^{-i E \ln p}\right)}{\left(1-\chi(p) c_{p} \mathrm{e}^{i E \ln p}\right)\left(1-\chi(p) d_{p} \mathrm{e}^{-i E \ln p}\right)} .
$$

As before, we introduce the diagonal approximation; taking into account that $\chi^{2}(p)=$ 1,0 , we obtain that $\left\langle z_{p}\right\rangle$ is given by Eq. (11) if $p$ is not a divisor of $k$, otherwise it drops out from the product $Z^{I}=\prod_{p}\left\langle z_{p}\right\rangle$. This implies that the part of the generating function obtained under the assumption $\operatorname{Im} e_{C, D}>0$ can be expressed as

$$
Z^{I}=Z_{\zeta}^{I}\left(e_{A}, e_{B}, e_{C}, e_{D}\right) \Phi^{I}\left(e_{A}, e_{B}, e_{C}, e_{D}\right) \Xi^{I}\left(e_{A}, e_{B}, e_{C}, e_{D}\right)
$$

with $Z_{\zeta}^{I}$ and $\Phi^{I}$ are the same as in Eqs. (1214). The function $\Xi^{I}$ cancels the factors now missing in $Z^{I}$, it can be expressed as a finite product over the prime divisors of $k$

$$
\Xi^{I}\left(e_{A}, e_{B}, e_{C}, e_{D}\right)=\prod_{p / k} \frac{1}{\left\langle z_{p}\right\rangle}=\prod_{p / k}\left(1+\frac{\left(b_{p}-d_{p}\right)\left(a_{p}-c_{p}\right)}{1-c_{p} d_{p}}\right)^{-1} .
$$

Differentiation of $Z^{I}$ with respect to $e_{A}$ and $e_{B}$ creates within the $(\|)$ - identification the non-oscillatory contribution to the correlation function as given in Ref.

[31. The oscillatory part is obtained as $\left(\partial_{e_{A} e_{B}}^{2} Z^{I I}\right)_{\|}$with $Z^{I I}\left(e_{A}, e_{B}, e_{C}, e_{D}\right)=$ $Z^{I}\left(e_{A}, e_{B},-e_{D},-e_{C}\right)$; it contains the factor additional to the Riemann zeta case

$$
\left.\Psi^{\mathrm{off}}(e) \equiv \Xi^{I}\left(e_{A}, e_{B},-e_{D},-e_{C}\right)\right|_{\|}=\prod_{p / k}\left(1+\frac{\left(p^{i e / 2}-p^{-i e / 2}\right)^{2}}{p-p^{-i e}}\right)^{-1}
$$

which was earlier found in Ref. [31] based on the Hardy-Littlewood conjecture. We observe that the correlation function depends only on the period $k$ but not on the precise form of the characters $\chi(n)$.

\section{Dynamical systems without time reversal; non-universal corrections}

In this section we return to dynamical systems with classically chaotic counterpart. We show how the results from Ref. [7] can be obtained by the method proposed here including the nonuniversal effect of repetitions.

The expression for the spectral determinant $\operatorname{det}(E-H)$ taking into account repetitions of periodic orbits is given by [2, 25]

$$
\operatorname{det}(E-H)=B(E) \mathrm{e}^{-i \bar{N}(E)} \prod_{p} \prod_{k=0}^{\infty}\left(1-\frac{\mathrm{e}^{i S_{p}(E) / \hbar}}{\Lambda_{p}^{k+1 / 2}}\right)
$$

expressed as product over the primitive periodic orbits $p$ with the actions $S_{p}(E)$ and the stability coefficients $\Lambda_{p}=\mathrm{e}^{\lambda_{p} T_{p}}$ depending on the Lyapunov exponent $\lambda_{p}$ and the duration of $T_{p}$ of the orbit $p$. The function $\bar{N}(E)$ is given in Eq. (5) and $B(E)$ again is a real function resulting from the regularization of the infinite product. 
New approach to periodic orbit theory of spectral correlations

Expanding again $S_{p}(E+e) \approx S_{p}(E)+e T_{p}, z_{p}$ is obtained in diagonal approximation as

$$
\left\langle z_{p}\right\rangle=\frac{1}{2 \pi} \int_{0}^{2 \pi} d \phi \frac{\left(\mathrm{e}^{i e_{C} T_{p}+i \phi} / \sqrt{\Lambda_{p}} ; \Lambda_{p}^{-1}\right)_{\infty}}{\left(\mathrm{e}^{i e_{A} T_{p}+i \phi} / \sqrt{\Lambda_{p}} ; \Lambda_{p}^{-1}\right)_{\infty}} \frac{\left(\mathrm{e}^{i e_{D} T_{p}-i \phi} / \sqrt{\Lambda_{p}} ; \Lambda_{p}^{-1}\right)_{\infty}}{\left(\mathrm{e}^{i e_{B} T_{p}-i \phi} / \sqrt{\Lambda_{p}} ; \Lambda_{p}^{-1}\right)_{\infty}}
$$

with the $q$-Pochhammer symbols defined as

$$
(x ; q)_{n}=\prod_{k=0}^{n-1}\left(1-x q^{k}\right) .
$$

The averaging of (27) with respect to $\mathrm{e}^{i \phi}$ is performed by the $q$-binomial formula of Gauss 35

$$
\frac{(t x ; q)_{\infty}}{(x ; q)_{\infty}}=\sum_{n=0}^{\infty} \frac{(t ; q)_{n}}{(q ; q)_{n}} x^{n}
$$

yielding

$$
\begin{aligned}
\left\langle z_{p}\right\rangle & =\frac{1}{2 \pi} \int_{0}^{2 \pi} d \phi \sum_{n, m=0}^{\infty} \frac{\left(\mathrm{e}^{i\left(e_{C}-e_{A}\right) T_{p}} ; \Lambda_{p}^{-1}\right)_{n}}{\left(\Lambda_{p}^{-1} ; \Lambda_{p}^{-1}\right)_{n}}\left(\frac{\mathrm{e}^{i \phi+i e_{A} T_{p}}}{\sqrt{\Lambda_{p}}}\right)^{n} \frac{\left(\mathrm{e}^{i\left(e_{D}-e_{B}\right) T_{p}} ; \Lambda_{p}^{-1}\right)_{m}}{\left(\Lambda_{p}^{-1} ; \Lambda_{p}^{-1}\right)_{m}}\left(\frac{\mathrm{e}^{-i \phi+i e_{B} T_{p}}}{\sqrt{\Lambda_{p}}}\right)^{m} \\
& =\sum_{n=0}^{\infty} \frac{\left(\mathrm{e}^{i\left(e_{C}-e_{A}\right) T_{p}} ; \Lambda_{p}^{-1}\right)_{n}}{\left(\Lambda_{p}^{-1} ; \Lambda_{p}^{-1}\right)_{n}} \frac{\left(\mathrm{e}^{i\left(e_{D}-e_{B}\right) T_{p}} ; \Lambda_{p}^{-1}\right)_{n}}{\left(\Lambda_{p}^{-1} ; \Lambda_{p}^{-1}\right)_{n}}\left(\frac{\mathrm{e}^{i\left(e_{A}+e_{B}\right) T_{p}}}{\Lambda_{p}}\right)^{n},
\end{aligned}
$$

where the last sum is the definition of the $q$-hypergeometric function of Heine ${ }_{2} \varphi_{1}\left(\mathrm{e}^{i\left(e_{C}-e_{A}\right) T_{p}}, \mathrm{e}^{i\left(e_{D}-e_{B}\right) T_{p}} ; 1 / \Lambda_{p} ; 1 / \Lambda_{p} ; \mathrm{e}^{i\left(e_{A}+e_{B}\right) T_{p}} / \Lambda_{p}\right)$ [36, 37]. In the same way as before in Eq. (12) we can factorize from $Z^{I}=\prod_{p}\left\langle z_{p}\right\rangle$ its poles and zeros such that the rest is a convergent product. Therefore we consider the inverse classical zeta functions

$$
\frac{1}{Z_{\mathrm{cl}}(s)}=\prod_{p} \frac{1}{Z_{\mathrm{cl}, p}(s)}=\prod_{p} \prod_{k=0}^{\infty}\left(1-\frac{\mathrm{e}^{s T_{p}}}{\Lambda_{p}^{k+1}}\right)^{k+1} .
$$

The component $Z^{I}$ of the generating function can then be written as

$$
\begin{aligned}
Z^{I}= & \frac{Z_{\mathrm{cl}}\left(i\left(e_{A}+e_{B}\right)\right) Z_{\mathrm{cl}}\left(i\left(e_{C}+e_{D}\right)\right)}{Z_{\mathrm{cl}}\left(i\left(e_{A}+e_{D}\right)\right) Z_{\mathrm{cl}}\left(i\left(e_{B}+e_{C}\right)\right)} \prod_{p}\left(\frac{Z_{\mathrm{cl}, p}\left(i\left(e_{A}+e_{B}\right)\right) Z_{\mathrm{cl}, p}\left(i\left(e_{C}+e_{D}\right)\right)}{Z_{\mathrm{cl}, p}\left(i\left(e_{A}+e_{D}\right)\right) Z_{\mathrm{cl}, p}\left(i\left(e_{B}+e_{C}\right)\right)}\right)^{-1} \\
& \times_{2} \varphi_{1}\left(\mathrm{e}^{i\left(e_{C}-e_{A}\right) T_{p}}, \mathrm{e}^{i\left(e_{D}-e_{B}\right) T_{p}} ; 1 / \Lambda_{p} ; 1 / \Lambda_{p} ; \mathrm{e}^{i\left(e_{A}+e_{B}\right) T_{p}} / \Lambda_{p}\right) \mathrm{e}^{-i \pi \bar{\rho}\left(e_{C}-e_{B}-e_{A}+e_{D}\right)} .
\end{aligned}
$$

For $e_{A, B, C, D} \rightarrow 0$

$$
\frac{1}{Z_{\mathrm{cl}}\left(i\left(e_{A}+e_{D}\right)\right) Z_{\mathrm{cl}}\left(i\left(e_{B}+e_{C}\right)\right)} \rightarrow-\left(e_{A}+e_{D}\right)\left(e_{B}+e_{C}\right) T_{0}^{2}
$$

with a certain reference time $T_{0}\left[2\right.$. The overall generating function is the sum $Z^{I}+Z^{I I}$ where $Z^{I I}$ is obtained by the Weyl substitution $e_{C} \rightleftarrows-e_{D}$. Then the spectral correlation function of [7] is obtained by Eq. (2). 


\section{Systems with time reversal}

If time reversal is allowed almost every periodic orbit exists in two versions with different sense of traversal and exactly the same action. Repeating the calculations of Section 3 we get the generating function in the diagonal approximation as $Z=Z_{\text {diag }}^{I}+Z_{\text {diag }}^{I I}$, where

$$
\begin{aligned}
& Z_{\text {diag }}^{I}\left(e_{A}, e_{B}, e_{C}, e_{D}\right)=e^{-i \pi\left(e_{C}+e_{D}-e_{A}-e_{B}\right) \bar{\rho}} \frac{\left(e_{A}+e_{D}\right)^{2}\left(e_{B}+e_{C}\right)^{2}}{\left(e_{A}+e_{B}\right)^{2}\left(e_{C}+e_{D}\right)^{2}}, \\
& Z_{\text {diag }}^{I I}\left(e_{A}, e_{B}, e_{C}, e_{D}\right)=Z_{\operatorname{diag}}^{I}\left(e_{A}, e_{B},-e_{D},-e_{C}\right) .
\end{aligned}
$$

The term $Z_{\text {diag }}^{I I} \propto\left(e_{A}-e_{C}\right)^{2}\left(e_{B}-e_{D}\right)^{2}$ does not contribute to the correlation function since its second mixed derivative by $e_{A, B}$ turns into zero in the columnwise limit. Therefore in the presence of the time reversal the oscillatory contribution to the correlation function is a truly off-diagonal effect different from the Hardy-Littlewoodlike mechanism; attempts to obtain it in the framework of the diagonal approximation were unsuccessful [7].

The off-diagonal corrections to the generating function of the orthogonal case were obtained in [13, 14]; the symplectic case was considered in [15]. Their existence is due to the so called orbit partnership existing in the chaotic motion whose significance was realized after the discovery of the Sieber-Richter pairs [8]. The result has the form of an asymptotic expansion valid for large scaled energy deviations $\varepsilon_{X}=$ $e_{X} 2 \pi \bar{\rho}, \quad X=A, B, C, D$. Here the $e_{X}$ are assumed to be small in classical terms while the dimensionless variables $\varepsilon_{X}$ are considered to be large. In the orthogonal case the generating function can be written as $Z_{\text {ortho }}=Z^{I}+Z^{I I}$ with

$$
\begin{aligned}
Z^{I} & =Z_{\text {diag }}^{I}\left(1+Z_{o f f}^{I}\right) \\
Z_{\text {diag }}^{I} & =e^{i\left(\varepsilon_{A}+\varepsilon_{B}-\varepsilon_{C}-\varepsilon_{D}\right) / 2} \frac{\left(\varepsilon_{A}+\varepsilon_{D}\right)^{2}\left(\varepsilon_{B}+\varepsilon_{C}\right)^{2}}{\left(\varepsilon_{A}+\varepsilon_{B}\right)^{2}\left(\varepsilon_{C}+\varepsilon_{D}\right)^{2}}, \\
Z_{\text {off }}^{I} & \sim-\frac{\left(\varepsilon_{A}-\varepsilon_{C}\right)\left(\varepsilon_{B}-\varepsilon_{D}\right)}{\left(\varepsilon_{A}+\varepsilon_{D}\right)\left(\varepsilon_{B}+\varepsilon_{C}\right)} \sum_{n=1}^{\infty} \frac{(-2 i)^{n}(n-1) !}{\left(\varepsilon_{A}+\varepsilon_{B}\right)^{n-1}}\left(\frac{1}{\varepsilon_{C}+\varepsilon_{D}}+\frac{n}{\varepsilon_{A}+\varepsilon_{B}}\right)
\end{aligned}
$$

and $Z^{I I}\left(\varepsilon_{A}, \varepsilon_{B}, \varepsilon_{C}, \varepsilon_{D}\right)=Z^{I I}\left(\varepsilon_{A}, \varepsilon_{B},-\varepsilon_{D},-\varepsilon_{C}\right)$. The corresponding expansion in the symplectic case is obtained by the substitution,

$$
Z_{\text {sympl }}\left(\varepsilon_{A}, \varepsilon_{B}, \varepsilon_{C}, \varepsilon_{D}\right)=Z_{\text {ortho }}\left(-\varepsilon_{C},-\varepsilon_{D},-\varepsilon_{A},-\varepsilon_{B}\right) \text {. }
$$

Differentiating the generating functions by $\varepsilon_{A, B}$ and then setting all arguments equal to $\varepsilon$ we get the correlation functions as a diverging series in $\varepsilon^{-1}$ which can be brought to a closed form by the Borel summation. This is essentially a term-by-term Fourier transform to the time domain followed by summation of the resulting converging series. Finally the inverse Fourier transform brings the result back to the energy domain. An outstanding feature of the symplectic case is that the asymptotic expansion of the resulting correlation function contains two oscillatory components proportional respectively to $e^{i 2 \varepsilon}$ and $e^{i \varepsilon}$, the latter not present in the input of the Borel summation; for explanation see [15] . 


\section{Conclusion}

We present here a new way to compute spectral correlation functions starting from semiclassical expressions. It is carried out via the auxiliary generating function which is an averaged ratio of four spectral determinants. The semiclassical asymptotics of the generating function consists of two terms responsible for the monotonic and oscillatory components of the correlator. Our new way is to explain this twofold structure by complexifying the arguments of the generating functions and bringing them to the boundaries of convergence of the Gutzwiller periodic orbit expansions of the spectral determinants. That could be done in two ways differing by the direction in which we move away from the real axis; in the example of the Riemann zeta zeros it would mean bringing the arguments of zetas in the numerator of the generating function either to the left or to the right border of the critical strip. In our approach after the energy averaging the two semiclassical approximations of the generating function become wellbehaved analytical functions dominant in the respective complex half-planes; at the real axis the asymptotics of the generating function is given by their sum. We avoid the use of the Riemann-Siegel-lookalike formula for the spectral determinants, so far not proven beyond the Riemann zeta and quantum maps in finite Hilbert space. We avoid thus the appearance of additional terms caused by the sharp cut off at $T_{H} / 2$ and disappearing in the final result. Our approach has the additional advantage of simplicity, e.g., our derivation of the correlation function of the Riemann zeta and $L$-functions zeros is probably the simplest existing.

In the absence of the time reversal the oscillatory contribution to the correlation function can be found via the diagonal approximation to the generating function totally neglecting the periodic orbit correlations. On the other hand, if we choose to directly calculate the correlator via the periodic orbit sum the oscillatory components would exist only if the action correlation between the periodic orbits is taken into account [26]; in the case of the Riemann zeta zeros this is the correlation of primes following from the Hardy-Littlewood conjecture. It is still not quite clear what is the nature of that correlation in physical systems and why it does not reveal its presence if time reversal is allowed. It is also not clear why the existence of that correlation automatically follows from the Weyl symmetry of the generating function.

\section{Acknowledgements}

We are grateful to F. Haake for remarks which stimulated the work on the subject. We also thank T. Guhr who read the manuscript and made useful comments and M. Akila, M. Bruckhoff for discussions.

\section{References:}

[1] H.-J. Stöckmann, Quantum chaos: an introduction, Cambridge University Press, Cambridge (2008). 
New approach to periodic orbit theory of spectral correlations

[2] F. Haake, Quantum Signatures of Chaos, Springer-Verlag, Berlin, (2010).

[3] M.C. Gutzwiller, Chaos in classical and quantum mechanics, Springer (1990).

[4] T. Guhr, A. Müller-Groeling, H. Weidenmüller, Random Matrix Theories in Quantum Physics: Common Concepts, Phys. Rep. 299, 189 (1998).

[5] O. Bohigas, M.J. Giannoni, C. Schmit, Characterization of Chaotic Quantum Spectra and Universality of Level Fluctuation Laws, Phys. Rev. Lett. 52, 1 (1984).

[6] M.V. Berry, Semiclassical Theory of Spectral Rigidity, Proc. R. Soc. London, 400, 229 (1985).

[7] E.B. Bogomolny, J.P. Keating, Gutzwiller's Trace Formula and Spectral Statistics: Beyond the Diagonal Approximation, Phys. Rev. Lett. 77, 1472 (1996).

[8] M. Sieber, K. Richter, Correlations between periodic orbits and their rôle in spectral statistics, Physica Scripta, 2001, 128 (2001).

[9] J. Bolte, S. Keppeler, Semiclassical Form Factor for Chaotic Systems with Spin 1/2, J. Phys. A 32, 8863 (1999).

[10] S. Heusler, The semiclassical origin of the logarithmic singularity in the symplectic form factor, J. Phys. A 34, L483 (2001).

[11] J. Bolte, J. Harrison, The spin contribution to form factor of quantum graphs, J. Phys. A 36, 2747 (2003).

[12] S. Müller, S. Heusler, P. Braun, F. Haake, A. Altland, Periodic-orbit theory of universality in quantum chaos, Phys. Rev. E 72, 046207 (2005).

[13] S. Heusler, S. Müller, A. Altland, P. Braun, F. Haake, Periodic-Orbit Theory of Level Correlations, Phys. Rev. Lett. 98, 044103 (2007).

[14] S. Müller, S. Heusler, A. Altland, P. Braun, F. Haake, Periodic-orbit theory of universal level correlations in quantum chaos, New J. Phys. 11, 103025 (2009).

[15] P. Braun, Beyond the Heisenberg time: semiclassical treatment of spectral correlations in chaotic systems with spin 1/2, J. Phys. A 45, 045102 (2012).

[16] T. Nagao, S. Müller, The n-level spectral correlations for chaotic systems, J. Phys. A 42, 375102, (2009).

[17] J.P. Keating, The semiclassical functional equation, Chaos 2, 15 (1992).

[18] J.P. Keating, S. Müller, Resummation and the semiclassical theory of spectral statistics, Proc. R. Soc. A 463, 3241 (2007).

[19] D. Waltner, S. Gnutzmann, G. Tanner, K. Richter, Subdeterminant approach for pseudo-orbit expansions of spectral determinants in quantum maps and quantum graphs, Phys. Rev. E 87, 052919 (2013).

[20] R. Band, J.M. Harrison, C.H. Joyner, Finite pseudo orbit expansions for spectral quantities of quantum graphs, J. Phys. A 45, 325204 (2012).

[21] E.C. Titchmarsh, The Theory of the Riemann Zeta-function, Oxford University Press, Oxford (1988).

[22] H.M. Edwards, Riemann's Zeta Function, Academic, New York, 1974.

[23] M.V. Berry, The Riemann-Siegel expansion for the zeta function: high orders and remainders, Proc. R. Soc. Lond. A 450, 439462 (1995).

[24] E.B. Bogomolny, Riemann Zeta Function and Quantum Chaos, Progress of Theo. Phys. Supp., 16619 (2007).

[25] J.P. Keating, The Riemann Zeta-Function and Quantum Chaology. in Quantum Chaos, G. Casati, I. Guarneri, U. Smilansky, eds., North-Holland, Amsterdam, pp. 145-185, (1993).

[26] N. Argaman, F.-M. Dittes, E. Doron, J.P. Keating, A.Yu. Kitaev, M. Sieber, U. Smilansky, Correlations in the Actions of Periodic Orbits Derived from Quantum Chaos, Phys. Rev. Lett. 71, 4326 (1993).

[27] H.L. Montgomery, The pair correlation of zeros of the zeta function, Analytic Number Theory (St. Louis, 1972), Proc. Sympos. Pure Math. 24, Amer. Math. Soc. (Providence), pp. 181-193, (1973).

[28] A.M. Odlyzko, The $10^{20}$-th zero of the Riemann zeta function an 70 million of its neighbors. 
New approach to periodic orbit theory of spectral correlations

Preprint, AT\& T Bell Laboratories, (1989).

[29] E.B. Bogomolny, J.P. Keating, Random matrix theory and the Riemann zeros I: three and fourpoint correlations, Nonlinearity 8, 1115 (1995).

[30] E.B. Bogomolny, J.P. Keating, Random matrix theory and the Riemann zeros II: n-point correlations, Nonlinearity 9, 911 (1996).

[31] E.B. Bogomolny, J.P. Keating, Two-point correlation function for Dirichlet L-functions, J. Phys. A 46, 095202 (2013).

[32] E.B. Bogomolny, J.P. Keating, A method for calculating spectral statistics based on random-matrix universality with an application to the three-point correlations of the Riemann zeros, J. Phys. A, 46, 305203 (2013).

[33] M.V. Berry, J.P. Keating, The Riemann zeros and eigenvalue asymptotics, SIAM Review 41, 236 (1999).

[34] J. B. Conrey, N. C. Snaith, Applications of the L-Functions Ratios Conjectures, Proc. London Math. Soc. 94, 594 (2007).

[35] Wolfram Math World: q-Binomial Theorem, http://mathworld.wolfram.com/qBinomialTheorem.html.

[36] Wolfram Math World: q-Hypergeometric Function, http://mathworld.wolfram.com/qHypergeometricFunction.html.

[37] E. Heine, Untersuchungen über die Reihe $1+\frac{\left(1-q^{\alpha}\right)\left(1-q^{\beta}\right)}{(1-q)\left(1-q^{\gamma}\right)} x+\frac{\left(1-q^{\alpha}\right)\left(1-q^{\alpha+1}\right)\left(1-q^{\beta}\right)\left(1-q^{\beta+1}\right)}{(1-q)\left(1-q^{2}\right)\left(1-q^{\gamma}\right)\left(1-q^{\gamma+1}\right)} x^{2}+\ldots$, J. reine angew. Math. 34, 285 (1847). 